\title{
Migration of a Bullet from the Inferior Vena Cava to the Right Pulmonary Artery : Case Report
}

\author{
Argun Saylam, M.D., A. Yüksel Bozer, M.D., \\ and Yilmaz KadioĞLU, M.D.
}

\section{Summary}

A case of early migration of a bullet from the inferior vena cava to the right pulmonary artery passing through the right heart, treated by right upper lobectomy, is presented. Pulmonary scanning, right heart catheterization and angiography are emphasized as valuable methods leading to diagnosis. World literature concerning foreign body migrations to the heart and pulmonary arteries by venous route are briefly discussed.

\section{Additional Indexing Words :}

Cardiac tamponade Embolism Endocarditis Foreign body embolism Haemopericardium Infection Sepsis

MIGRATION of foreign bodies from systemic veins to the heart or pulmonary arteries are rare in the world literature. To date about 26 cases of bullet emboli') and 38 cases of polyethylene catheter $\mathrm{emboli}^{21}$ have been reported in the literature. A rare case of bullet embolism to the right pulmonary artery following gunshot wound in the abdomen is the subject of this report.

\section{CASE Report}

A 14-year-old white boy (case number: 338563) was referred to our clinic on 3rd of June, 1972 by the consulting surgeons in Ankara Numune Hospital because of a bullet in the right lung. Patient's history revealed that he was shot accidentally in the right upper abdominal quadrant about 55 days ago. He was transferred to Ankara Numune Hospital, where he underwent an upper median laparotomy. Duodenum was found ruptured and sutured primarily. Vena cava inferior was also ruptured and repaired with sutures. Diaphragm was intact. No bullet could be found in the abdominal cavity. On a routine chest X-ray a bullet was detected in the right lung although the patient was not shot in the chest.

The patient had no complaints on admission to our hospital. Auscultation of both lungs revealed normal findings. No abnormality could be detected during his physical examination. He had no fever, a pulse of $94 / \mathrm{min}$, and blood pressure $110 / 70 \mathrm{~mm} . \mathrm{Hg}$. Blood examination showed a normal hemogram. Direct postero-

From the Departments of Adult Thoracic and Cardiovascular Surgery of Hacettepe University Hospitals and General Surgery of Ankara Numune Hospital, Ankara-Turkey.

Received for publication July 11, 1972. 
Vol. 13
No. 6

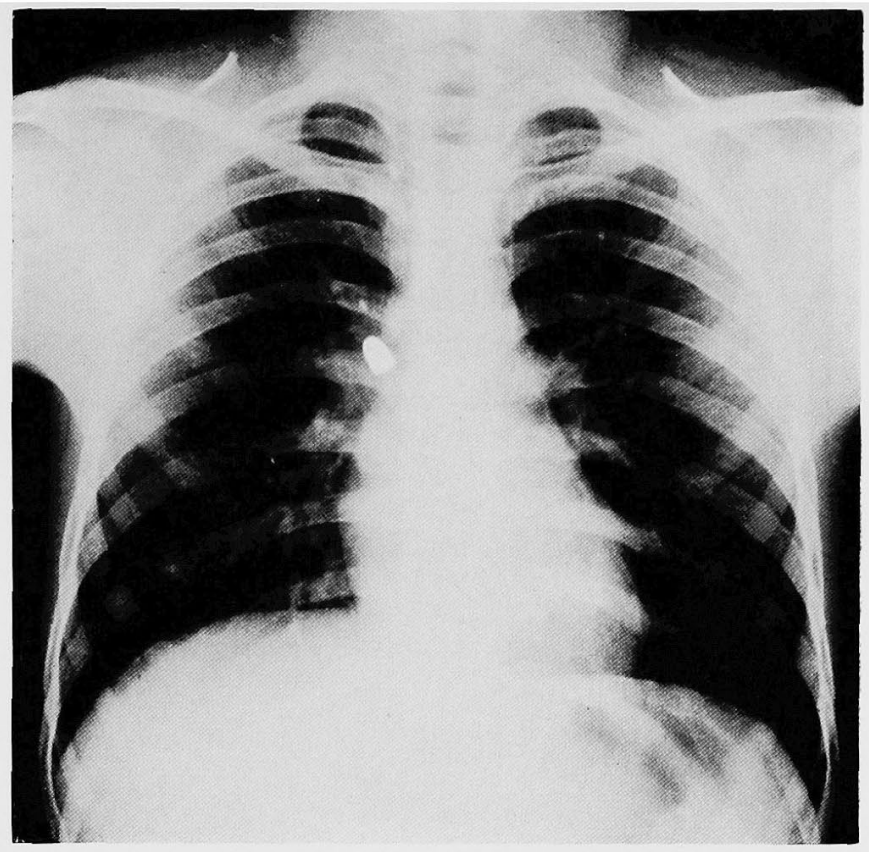

Fig. 1. Postero-anterior direct chest X-ray showing the bullet in the upper part of the right lung.

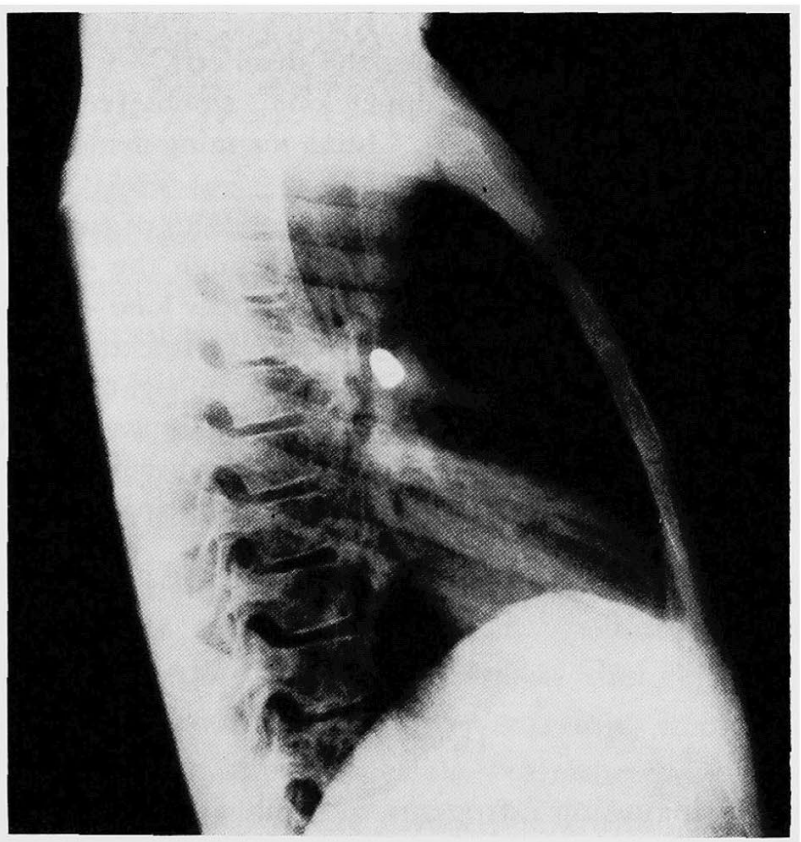

Fig. 2. Right-lateral direct chest X-ray demonstrating the bullet. 


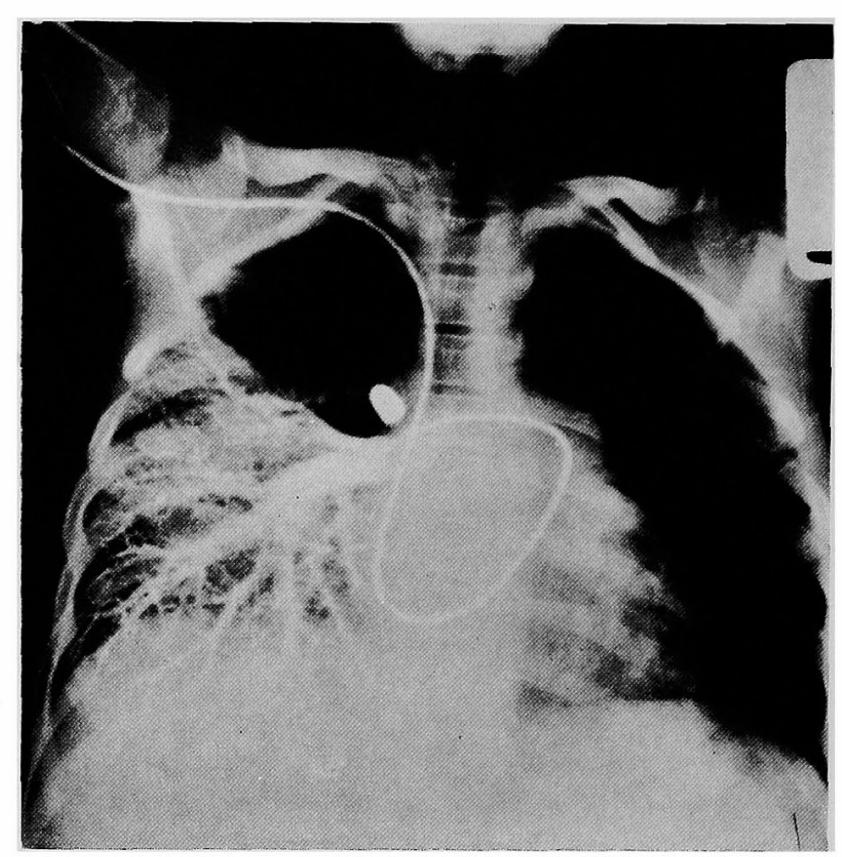

Fig. 3. Pulmonary angiography displaying the bullet in the pulmonary arterial branch of the right upper lobe. Notice the loss of perfusion in the affected lobe.

anterior and right lateral chest X-rays displayed a bullet probably located in the right pulmonary artery (Figs. 1 and 2). Right heart catheterization and angiocardiography were performed to ascertain the diagnosis. A bullet located in the pulmonary arterial branch of the right upper lobe was observed and perfusion was absent in the affected lobe (Figs. 3 and 4). Lung scanning demonstrated a cold area in the upper portion of the right lung.

The patient was taken to the operating room on 20th of June, 1972 and he was subjected to a right postero-lateral thoracotomy through the 4th intercostal space. Branch of the pulmonary artery entering the right upper lobe was prepared and the bullet felt was removed with pulmonary arteriotomy. Infection was present in the pulmonary artery, the neighboring bronchus and in the right upper lobe. Pulmonary back-flow was not satisfactory. Lymphadenomegaly was observed in the hilar region. Right upper lobe was removed and the right phrenic nerve was sectioned. Thoracotomy incision was closed in layers by placing apical and basal thoracic drainage tubes.

Postoperative course was uneventful. He was discharged from hospital on 1st of July with complete recovery.

\section{Discussion}

Foreign bodies including iatrogenic materials entering venous circulation by penetrating gastrointestinal tract or skin can migrate in sytemic venous 


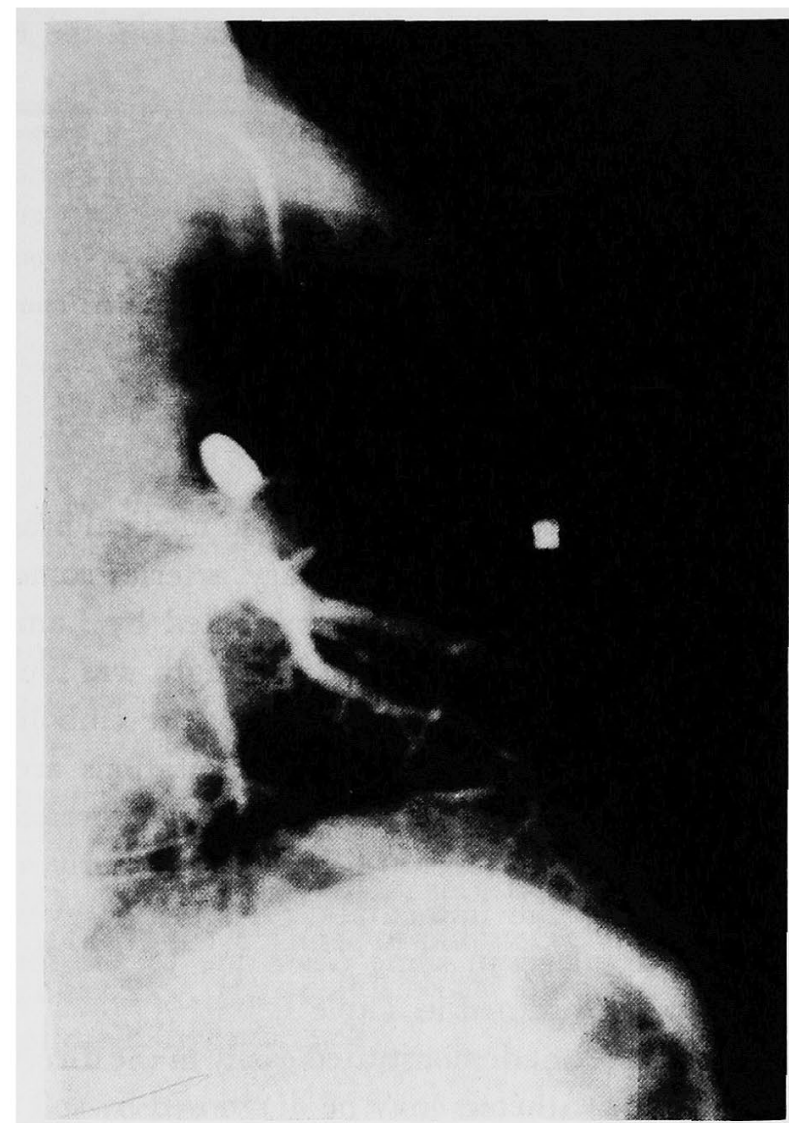

Fig. 4. Right-lateral view of the pulmonary angiography.

circulation to the right heart or pulmonary artery, among which needles, fragments of polyethylene catheters, nails, pins, pieces of zipper, pieces of wood, fish bones, hairpins, toothpicks, bullet and shrapnel are the encountered ones.1,3),4) First case of a bullet embolism was reported by Simons in 1903 as quoted by one of the authors and Hufnagel, ${ }^{4)}$ where they listed 18 cases up to 1962. Samaan ${ }^{3)}$ cited an interesting case reported by Barret in 1950, mentioning a girl who swallowed a hair grip penetrating through the pharynx to the systemic veins and then entering the right ventricle. One of the authors of this article and Hufnagel ${ }^{4}$ stated another very interesting case presented by Bailey in 1913, where a swallowed toothpick perforated the duodenum and passed into inferior vena cava migrating to the right atrial appendix. Migration of the foreign bodies in the lower extremity is sometimes prevented by venous valvulae of the affected vessels. ${ }^{5}$ ) Another way of entrance of the foreign bodies into the heart is the direct penetration through the chest or abdomen as seen in the wartime and civilian practice occurring either accident- 
Table I. Complications of the Foreign Bodies in the Heart and Pulmonary Arteries

1. Embolism :

a. of the foreign body itself

b. of the intracardiac thrombus

2. Intracardiac thrombus formation

3. Perforation of the heart wall, hemopericardium and cardiac tamponade

4. Infection and/or endocarditis :

a. bacterial

b. fungal (candidiasis etc.)

ally or on purpose. Such causalties are not rare in medical practice.

Foreign bodies in the heart or pulmonary arteries sometimes have no symptoms. Wellman et al. ${ }^{5}$ stated a case presented by Lamprecht in 1965 where a venous polyethylene catheter $40 \mathrm{~cm}$. in length was found in the right heart accidentally during necropsy 6.5 years after the embolism. Dyspnea, tachypnea, tachycardia, pain and neurotic manifestations are seen in some cases. A systolic murmur may be auscultated when the foreign body is in the pulmonary artery. ${ }^{6}$ Symptoms occur either in the early or late period following embolism, during the immediate 24 hours or even after years. ${ }^{5}$ Complications may take place in some cases due to the foreign material. Probable complications are outlined in Table $\mathrm{I}^{3 /-5)}$

Non-opaque bodies are not demonstrated easily in the direct X-rays. Such materials like polyethylene catheters may be diagnosed by soft tissue roentgen techniques or in the angiography displaying a filling defect due to the space occupying foreign body. ${ }^{5}$ Opaque bodies are seen easily both in the direct chest X-rays and cardio-angiography. Lung scanning is of great help and a simple method leading to diagnosis of foreign bodies embedded in the pulmonary artery, showing a cold area in the affected lobe as proved in our case.

Removal of the foreign bodies is the treatment of choice as approved by most of the authors because of the occurrence of possible fatal complications like sepsis and embolism, although some believe that they should not be removed unless symptoms exist. Patient may succumb to myocardial damage and bleeding early after the embolism, where septic complications account for the late deaths. Methods used for the removal of foreign bodies from the heart and pulmonary arteries are tabulated in Table II.1),2),4-6)

Non-operative treatment with special flexible endoscopic catheter like forceps with wire-looped tip is reported in the literature. ${ }^{2), 51}$ Normothermic or hypothermic inflow occlusion is used by some surgeons to remove the foreign bodies embedded in the right atrium or right ventricle near the tricuspid annulus. ${ }^{6}$ ) Open-heart surgery with extracorporeal circulation is a safe 
Table II. Management of the Foreign Bodies in the Heart and Pulmonary Arteries

1. Non-operative treatment:

Extraction of the foreign body with special fexible endoscopic forceps introduced through systemic veins

2. Operative treatment :

A. Foreign body located in the heart:

a. removal of the foreign body through the right atrium by inflow occlusion with/without hypothermia

b. open-heart surgery using extracorporeal circulation

B. Foreign body located in the pulmonary artery:

a. pulmonary arteriotomy

b. segmentectomy or lobectomy

method to remove the foreign bodies located in the cardiac chambers. Such a case, where a bullet was removed from the right ventricular apex first time with the help of extracorporeal circulation belongs to one of the authors of this paper and Hufnagel ${ }^{4)}$ in 1962.

Pulmonary arteriotomy is performed when the foreign body is in the pulmonary artery. Segmentectomy or lobectomy may be done when the attacked portion of the lung is infected or pulmonary back-flow is not satisfactory as happened in our case. Hence, it is no use to keep a non-functional and infected portion of the lung in the body.

\section{REFERENCES}

1. Padula, R. T., Sandler, S. C., Camishion, R. C.: Ann. Surg. 169: 599, 1969.

2. Graham, K.J., Barratt-Boyes, B. G., and Cole, D. S.: Brit. J. Surg. 57: 184, 1970.

3. Samaan, H. A.: Brit. J. Surg. 57: 685, 1970.

4. Bozer, A. Y. and Hufnagel, C. A.: Acta Medica Turcica 111: 186, 1966.

5. Wellman, K. F., Reinhard, A., and Salazar, E. P.: Circulation 37: 380, 1968.

6. Gschnitzer, F. and Wykypiel, H.: Chirurg 41: 88, 1970. 